\title{
Ocorrência de Cryptosporidium spp. e outros parasitas em hortaliças consumidas in natura, no Recife
}

\author{
Occurrence of Cryptosporidium spp. \\ and others parasites in vegetables consumed \\ in natu ra, Recife, Brazil
}

Celiane Gomes Maia da Silva 1

Samara Alvachian Ca rdoso Andrade 1

Tânia Lúcia Mon tenegro Stamford 1 *

$1^{\star}$ Departamento de Nutrição, Cen tro de Ciências da Saúde, UFPE. Rua Jader de Andrade 335, Casa Forte, 52061-060, Recife PE.

tlmstamford@yahoo.com.br
Abstract The study was carried with the aim to evalu a tethe occurren ce of en teropa rasites in veget ables commerialized and consumed in natural form in the state of Pernambuco, Brazil. Horticultural samples purchased from supermarket and free market: 40 from lettuce (Lactuca sativa), 40 from watercress (Nasturtium officinale) and 20 from chard (Beta vulgaris) were analyzed. Cryptosporidium spp. detection was realized following Mo nge and Arias methodology, using two staining processes (Koster modified and Ziehl$\mathrm{Ni}$ elsen). Parasitological analysis was determined by the spontaneous sedimentation te chnique (Gelli et al.), and total coliformes and Esch eri chia coli following And rews. The distribution of parasitic contamination was let tuce $60 \%$, wa tercress $30 \%$ and chard 20\%, with evidence of helminthes (Ascaris lumbricoides, Strongyloides stercoralis, Ancylostoma duodenale) and protozoas (Co mplex Entamoda histo lytica/Entamoeba dispar, Crypto sporidium spp. and Entamoeba coli). Lettuce showed greater contamination of total coliformes and Escherichia coli in samples from supermarket and watercress in samples from free market. These data suggest the need of adoption of educative programs for horticulturalists and monitoration of the water used in horticulture was in evidence as future needs.

Key words Crypto spori dium spp., Escherichia coli, Enteroparasites, Vegetables, Water
Resumo O objetivo deste estudo foi verificar a ocorrência de enteroparasitas em hortaliças comercializadas e con sumidas em Pernambuco. Foram utilizadas 100 amostras de hortaliças: 40 amostras de alface lisa (Lactuca sativa), 40 de agrião (Nasturtium officinale) e 20 de acelga (Beta vulgaris), proven ien tes de fei ras livres e supermercados. A deteç̧ão de Cryptosporidium s pp. foi realizada con forme Mo nge e Arias sendo utilizado dois métodos de coloração, Koster modificado e Ziehl-Nielsen. Foi usada a técnica de sedimentação espontânea de Gelli et al. para a análise parasitológica. As análises de coliformes totais $e$ Esch erichia coli foram realizadas de acordo com Andrews. Os resultados obtidos mostraram um percentual de contaminação parasitária em $60 \%$ de alface, $30 \%$ de agrião e $20 \%$ de acelga, destacando-se o Asc aris lumbricoides, Strongyloides stercoralis e Ancylostoma duodenale dentre os helmintos, e o Cryptosporidium spp., Entamoeba coli e o complexo Ent am oeba histo lytica/Entamoba díspar, dentre os protozo á rios com maior freqüência. As hortaliças mais contaminadas por col ifo rmes totais $e$ E sch erichia coli foram alfacenas amostras de su perm ercado e agrião em fei ra livre. Esses dados sugerem a necessidade da adoção de medidas educativas aos produtores, $e$ do monito ramento das águas destinadas à irrigação das hortas.

Palavras-ch ave Cryptosporid ium spp., Esch erichia coli, Enteroparasitas, Hortaliças, Água 


\section{Introdução}

Os vegetais são amplamente recomendados como parte da alimentação diária por seu apreciável conteúdo em vitaminas, sais minerais e fibras alimentares. Tem crescido o interesse, principalmente, por aqueles que apresentam em sua com posição substâncias com atividade antioxidante, a exemplo dos caro tenóides, vitamina $\mathrm{C}$ e flavonóides, que os caracterizam como alimentos funcionais.

As doenças transmitidas por alimen tos são resultantes predominantemente do ciclo de contaminação fecal/oral e seu con trole tem recebi do cada vez maior atenção em todo o mu ndo. Geralmente, tanto nas áreas rurais quanto urbanas dos países de Terceiro Mundo devido às más condições sanitárias, as parasitoses intestinais são amplamen te difundidas, sendo as hortaliças citadas como um dos veículos de suas estruturas infectantes (Slifkoet al., 2000). A principal forma de contaminação dessas hortaliças se dá pela água contaminada por material fecal de origem humana, utilizada na irrigação das hortas. Vá rios autores men cionam a possibilidade de transmissão de parasitoses ao homem por meio da ingestão de frutas e verduras con sumidas cruas, proveni en tes de áreas cul tivadas contaminadas por dejetos fecais (Si lva et al., 1995; Me s quita et al., 1999; Takayanagui et al., 2000; 2001).

O diagnósti co laboratorial de parasitas de ocorrência em humanos presen tes em hortaliças é de grande importância para a saúde pública uma vez que forn ece dados sobre as condições higiênicas envolvidas na produção, armazenamento, transporte e manuseio desses produtos e, portanto sobre os riscos de contaminação dos seus con su midores.

Dentre tantos parasitos que infectam o homem, o Cryptosporidium spp. atualmen te se a pre senta como um patógeno oportunista freqü en te principalmente em pacientes imunodeprimidos. Este para si to cosmopolita de caráter zoon ó ti co é en con trado em todos os con tin entes, tanto em países desenvolvidos como em desenvolvimento nas áreas urbanas e rurais ( $\mathrm{L}$ ima, 2001; Si lva et al., 2002; Lima \& Stamford, 2003).

Diversos trabalhos têm sido realizados para pe s quisar en teroparasitas em hortaliças, porém pou cos têm enfatizado a contaminação destas com o Crypto sporidium spp. É de ex trema rel evância a pesquisa deste para sito em virtu de de sua importância na saúde pública e, principal- mente, pelo grande impacto da criptosporidiose em paci en tes imu odeprimidos.

O pres en te estu do teve como obj etivo verificar a ocorrência de Cryptosporidium spp. e o utros en teroparasitas em hortaliças comercializadas na região metropolitana do $\operatorname{Recife}(\mathrm{PE})$, além de avaliar a contaminação por coliformes totais e Escherichia col i.

\section{Métodos}

Foram utilizadas cem unidades de hortaliças distri buídas em 40 amostras de alface lisa ( $L a c$ tuca sativa), 40 de agrião (Nasturtium officinale) e 20 de acel ga (Beta vulgaris) en tre os meses de maio a julho. Do total de hortaliças coletadas, $50 \%$ foram provenien tes de três diferentes fei ras livres e $50 \%$ de três diferen tes supermerc ados, a mbos localizados na região metropolitana do Recife.

Durante a coleta, as hortaliças foram acondicionadas, in dividualmente, em sacos plásticos e levadas ao laboratório para serem analisadas.

\section{Prep a ro das amostras}

As folhas das hortaliças foram selecionadas, excluindo-se as danificadas, e pesadas antes de s erem subm etidas à lavagem com água destilada para retirada de sujidades. Posteriormente, foram process adas pela técnica de lavagem de scrita por Monge e Arias (1996), onde $50 \mathrm{~g}$ de folhas foram lavadas com $450 \mathrm{ml}$ de solução salina $(\mathrm{NaCl} 0.85 \%)$, esfregando-se com pincel chato $\mathrm{n}^{\circ} 16$ num recipien te plásti co e dei xadas em repouso por 5 minutos. A seguir as folhas fora $\mathrm{m}$ desprezadas e a solução de lava gem submetida às análises microbiológicas e parasitológicas.

\section{Análises microbiológicas}

Foram utilizadas placas PetrifilmTM para contagem de coli formes totais e Escherichia coli de acordo com a metodologia de Andrews (1993).

\section{Pesquisa de Cryptosporidium spp.}

\section{- Prepa ro das lâminas}

Um vo lume de $50 \mathrm{ml}$ da solução de lava gem foi submetido à centrifugação a $900 \mathrm{rpm}$ por 30 minutos (Mon ge \& Arias, 1996). Para con- 
fecção dos esfregaços foi utilizado um volume de $20 \mathrm{ml}$ do sedim en to coletado após cen trifugação, em triplicata, num total de $60 \mathrm{ml}$ por amostra. Todas as lâminas foram confeccionadas em duplicata para aplicação de dois métodos de coloração, resultando em 200 lâminas com 600 esfregaços. Através de sucção com pipeta automática os sedimentos foram medidos ten do em média $0,25 \mathrm{ml}$ de sed i $\mathrm{m}$ en to final por amostra.

\section{Processo de coloração}

Pa ra a coloração dos esfregaços foram utilizadas as técnicas de Koster modificada (Ka geruka et al., 1984) e a técnica de Ziehl-Niels en (Luna, 1968) modificada pelo uso da fucsina fenolada.

\section{Leitura e contagem dos oocistos}

A leitu ra e a contagem dos oocistos foram realizadas utilizando-se microscopia óptica com obj etiva de imersão, percorren do-se todo o esfregaço. Observou-se a característica dos oocistos referen te à forma, cor e estruturas internas em ambos os métodos de coloração (Ongerth \& Stibbs, 1987; Bennet et al., 1999).

Para o cálculo do número total de oocistos na amostra utilizou-se a metodologia descrita por Ol ivei ra \& Germano (1992a).

\section{Pesquisa de en teroparasitas}

O restante da solução de lavagem passou por um processo de sedimentação em cálices cônicos du ran te 24 horas. Pa ra cada amostra foram utilizados três esfregaços com cerca de $1 \mathrm{ml}$ do sedimento cada, posteriormente corado com lu gol e analisado ao microscópio óptico, i nve stiga ndo-se a pre sença de formas contaminantes de enteroparasitas (Gelli et al., 1979).

\section{Análise estatística}

Os dados foram avaliados através da análise de variância (ANOVA), s en do aplicado o Teste de Tuckey e a correlação de Pearson (r) no nível de 5\% de significância. As análises estatísticas foram realizadas através do programa Stati stica Soft for Windows 6.0.

\section{Resultados e discussão}

Os percen tuais de contaminação por en terop arasitas nas três varied ades de hortaliças estudadas foram $60 \%$ para alface lisa, $30 \%$ para agrião e $20 \%$ para acelga. Vale ressaltar que o Crypto sporidium ssp. foi detectado apenas nas amostras de alface lisa, portanto o percentual de contaminação, excluindo-se este parasita, foi de 30\% neste vegetal. Através desses resultados observamos que apesar das limitações dos métodos empregados, o nível de contaminação encon trado foi bastante el evado.

Takayanagui et al. (2001), analisando 139 amostras de alface, detectaram 33\% de contaminação parasitária, resultado con si derado semelhanteao nosso, quando comparado às amostras sem os valores de Cryptosporidium spp. Guilherme et al. (1999) na cidade de Maringá (PR) con stata ram 21,4\% de contaminação parasitária para alface lisa e $100 \%$ para agrião, enqu a n to que Ol ivei ra \& Germano (1992a, 1992b) eviden c i a ramem São Paulo vários tipos de helmintos em $32 \%$ das amostras de alface lisa e em $66 \%$ de agrião, e na pesquisa de protozoários detectaram $18 \%$ e $60 \%$, respectivamente. Es tes resultados con trastam com os baixos níveis de contaminação por estruturas parasitárias relatados nas cidades de Niterói e Rio de Janeiro (RJ), onde apenas 3,9\% de alface e $2,3 \%$ de agrião apresentaram contaminação por en teroparasitas. Os autores sugerem que esses resultados, possivelmente, indicam uma melhoria na qualidade higiênica no plantio, i rrigação, armazen a gem e distribuição de hort aliças (Me s quita et al., 1999).

É possível que a estrutura física natural das h ortaliças estu d adas con tribua para a ocorrência de diferenças nos percen tuais de contaminação ob s ervados en tre as três vari ed ades. Isto se aplica, s obretu do, aos re sult ados obti dos em rel a ção à alface e ao agrião, que se mostram maiores que os obti dos para acel ga. O agrião, por po s suir folhas múltiplas e separadas, permite maior fixação dos cistos. A alface possui folhas largas, ju sta postas e flexíveis poden do ocorrer contato com o solo du rante o cultivo levan do a um maior índice de contaminação, depen den do das condições de cultivo. A acelga assemelha-se à alface por po s suir folhas largas e justapostas, porém rígidas, prom oven do ao vegetal uma forma fechada e firme que dificulta seu con t a tocom o solo, diminuindosua carga decontaminação.

No nosso ex perimen to, a acel ga apresentou um nível de contaminação de $20 \%$, s en do a hor- 
taliça que mostrou menor percentual entre as amostras estudadas. Não foram encontrados na literatura estu dos abord a ndo a contaminação da acelga por enteroparasitas, não permitindo comparações.

A análise das freqüências de cada tipo de en teroparasita (Ta bela 1) mostrou predom inância de protozoários no total de amostras analisadas ( complexo Entamoeba histolytica/Entamoeba dispar (14,0\%), Cryptosporidium spp. $(12,0 \%)$ e Entamoeba coli (10,0\%) em comparação com os helmintos (Ascaris lumbricoides (5,0\%), Strongylo ides sterco ralis (3,0\%) e Ancylostoma duodenale $(3,0 \%))$. Entre os enterop arasitas encon trados com menor freqüência incluíram-se Giárdia lambia (1,0\%), Trich uris trichuras (1,0\%) e Hymenol epis nana (1,0\%). A men or freqüência de enteroparasitas foi detec$\mathrm{t}$ ada nas amostras de acel ga .

Devido à dificuldade de fazer a distinção por micro scopia entre Entamoeba histolytica e Entam oeba dispar, estas foram dassificadas como Complexo Entam oeba histolytica/Entamoeba dispar, ressaltando que Entamoeba histolytica provoca doença intestinal conhecida como amebíase e a Entamoeba díspar não causa doença (Gomes et al., 1999; Freitas et al., 2004).

De acordo com diversos autores, as condições sanitárias do ambien te em que as hortaliças são cultivadas, as práticas de cultivo utilizadas e a sua estrutura física possivelm en te justificam as diferenças entre os percentuais de contaminação nas variedades de hortaliças (Mesquita et al., 1999; Coelho et al., 2001; Takayanagui et al., 2001). Deste modo, os menores percentuais de contaminação observados nas a mostras de acel ga podem ser atribuídos, en tre o utros fatores, às suas melhores condições de cultivo. Diversos estudos comprovam que os ovos de helmintos podem sobreviver por períodos de tempo mais prolongados no meio aquático (Slifko et al., 2000). Este fato poderia justificar a freqüência encontrada no agrião, cujo cultivo exige terrenos permanen temente úmidos.

Com relação aos enterop arasitas, todos são de importância para a saúde pública. A maioria indica contaminação fecal de ori gem humana e/ou animal, tal como ocorre com Ascaris lumbricoides, Trichuris trichuras, Hymenolepis nana, Strongyloides sterco ralis e Ancylostoma duodenale, uma vez que acom etem o hom em e o utros animais (Cim erman et al., 1999; Slifko et al., 2000). Há, portanto, similaridade entre es tes resultados e os ob servados por outros au- tores no país quando relacionados à contaminação por en terop arasitas em vegetais (Oliveira \& Germano, 1992a; 1992b; Guilh eme et al., 1999; Ta kayanagui et al., 2000), embora tenha ocorri do va riação na freqüência das espécies de parasitas qua ndo comparada aos trabalhos ci$\mathrm{t}$ ados anteri ormente.

Alguns autores consideram, no entanto, que a ordem de freqüência dos enteroparasitas nas hortaliças não é necessariamente a mesma en contrada na população humana do local estudado, devido, sobretudo, às diferenças na c a rga parasitária e na eliminação diária dos ovos pelos hospedeiros, variá veis para cada tipo de pa rasita (Rey, 1991; Slifkoet al., 2000).

É provável que a contaminação das hortaliças por oocis tos de Crypto sporidium spp. e outros parasitas seja provenien te, principalmente, das condições sanitárias do ambien te em que são cultivadas, das práticas de cultivo, da quali$\mathrm{d}$ ade da água utilizada tanto na irrigação quanto na higi enização antes de serem comerciali$\mathrm{z}$ adas em su permerc ados. Seg un doKori ch et al. (1990) o Cryptosporidium spp. é resisten te ao tratamen to conven ci onal de águas de abastecimen to utilizando-se cloro.

As médias das contagens de coliformes totais e de Escherichia coli obtida nas amostras analisadas en con tram-se na tabela 2. Com relação à con ta gem de coli formes totais nas amostras proven i en tes de su perm ercados, ho uve diferença significativa en tre alface e agrião. Nas amostras provenientes de fei ras livres houve diferença significativa entre alface e acelga e entre agrião e acel ga. O mesmo não foi constatado na contagem de Escherichia coli em nen huma das amostras provenien tes de supermercados, porém nas amostras oriundas de feira livre, observamos uma diferença significativa $(\mathrm{p}<0,05)$ en tre as amostras de agrião e acelga. Os dados obti dos revel a ram que as amostras de alface adquiridas de supermercados foram as mais contaminadas tanto para coliformes totais como E. coli qua ndo comparadas às outras hortaliças e nas amostras de feiras livres a mais contaminada foi a do agrião.

En tre as localidades só houve diferença significativa nas amostras de agrião, tanto para a população de coliformes totais como para a de E. coli (Tabela 2). Es tes dados mostram que a higi enização aplicada às hortaliças antes de est a rem ex postas à venda nos su permerc ados não é eficaz na diminuição de bactérias contaminantes em vegetais como alface e acelga. Porém, e s te proced im en to dem on strou eficiência 
Tabela 1

Freqüência e percen tual de enterop a rasitas por tipo de hortaliça estudada.

\begin{tabular}{|c|c|c|c|c|c|c|c|c|}
\hline \multirow[t]{2}{*}{ Espécie de Enteroparasita } & \multicolumn{2}{|c|}{$\begin{array}{l}\text { Alface lisa } \\
\quad \mathrm{N}=40\end{array}$} & \multicolumn{2}{|c|}{$\begin{array}{c}\text { Agrião } \\
\mathrm{N}=40\end{array}$} & \multicolumn{2}{|c|}{$\begin{array}{c}\text { Acelga } \\
\mathrm{N}=\mathbf{2 0}\end{array}$} & \multicolumn{2}{|c|}{$\begin{array}{c}\text { Total } \\
\mathrm{N}=100\end{array}$} \\
\hline & $\mathrm{F}$ & $\%$ & $\mathrm{~F}$ & $\%$ & $\mathrm{~F}$ & $\%$ & $\mathrm{~F}$ & $\%$ \\
\hline${ }^{\star}$ Crypto spo ridium spp. & 12 & 30,0 & 0 & - & 0 & - & 12 & 12,0 \\
\hline Entamoeba coli & 1 & 2,5 & 8 & 20,0 & 1 & 5,0 & 10 & 10,0 \\
\hline $\begin{array}{l}\text { Complexo Entamoba histolytical } \\
\text { Entamoeba díspar }\end{array}$ & 7 & 17,5 & 4 & 10,0 & 3 & 15,0 & 14 & 14,0 \\
\hline Giardia lambia & 0 & - & 1 & 2,5 & 0 & - & 1 & 1,0 \\
\hline Trichuris trichiura & 1 & 2,5 & 0 & - & 0 & - & 1 & 1,0 \\
\hline Ascaris lumbricoides & 2 & 5,0 & 3 & 7,5 & 0 & - & 5 & 5,0 \\
\hline Strongyloides sterco ralis & 1 & 2,5 & 1 & 2,5 & 1 & 5,0 & 3 & 3,0 \\
\hline Hymenolepis nana & 1 & 2,5 & 0 & - & 0 & - & 1 & 1,0 \\
\hline Ancylostoma duodenale & 0 & 0 & 3 & 7,5 & 0 & 0 & 3 & 3,0 \\
\hline
\end{tabular}

* Valor obti do combinando-se os resultados das duas metodologias investigad as.

$\mathrm{N}=$ número de amostras

$\mathrm{F}=$ freqüência

Tabela 2

Médias da contagem e desvio padrão de coliformes totais e Escherichia coli nas amostras oriundas de diferen tes localidades.

\begin{tabular}{|c|c|c|c|c|c|c|}
\hline & \multicolumn{6}{|c|}{ Localidades } \\
\hline & \multicolumn{3}{|c|}{ Su perm erc ados } & \multicolumn{3}{|c|}{ Feiras livres } \\
\hline & Alface lisa* & Agrião* $^{*}$ & Acelga $^{* *}$ & Alface lisa* & Agrião $^{*}$ & Acelga ${ }^{* *}$ \\
\hline Coliformes totais (UFC/g) 1 & $7,5 \mathrm{ac} \pm 0,6$ & $6,1 \mathrm{~b} \pm 1,4$ & $6,3 \mathrm{bc} \pm 1,4$ & $7,5 \mathrm{ac} \pm 0,8$ & $8,08 \mathrm{a} \pm 1,3$ & $5,3 \mathrm{~b} \pm 1,4$ \\
\hline Escherichia coli (UFC/g) 1 & $1,0 \mathrm{ab} \pm 1,4$ & $0,3 \mathrm{~b} \pm 0,9$ & $0,6 \mathrm{bc} \pm 1,9$ & $1,7 \mathrm{ac} \pm 1,3$ & $2,4 \mathrm{a} \pm 2,2$ & $0,0 \mathrm{bc} \pm 0,0$ \\
\hline
\end{tabular}

* Média de 20 amostras com três repetições.

** Média de 10 amostras com três repetições.

${ }^{1}$ Média da con t a gem representada pela função "Logaritmo Neperiano (LN)".

Na mesma linha letras iguais não diferem estatisticamen te ao nível de $5 \%$.

nas amostras de agrião on de aquelas provenien tes de su perm erc ados apresentaram resultados mais baixos tanto para coliformes totais como para E. col i.

Um percen tual de contaminação de $100 \%$ pa ra coliformes totais foi verificado nas amostras analisadas e foi confirmada a pres ença de Esch erichia coli em $50 \%$ das amostras de alface, $37,5 \%$ de agrião e $5 \%$ de acelga. Estes resultados dem onstram um grau de contaminação semelhante aos verificados para os en terop a rasitas citados anteriormente. Mon ge et al. (1996) detectaram $50 \%$ de amostras de alface contaminadas com coliformes totais on de em $42 \%$ foi confirmada a presença de Escherichia coli. Takayanagui et al. (2000), numa fiscalização de hortas produtoras de verdu ras no município de Ribeirão Preto (SP), detectaram a pre sença de elevada população de coliformes fecais em 22 das 129 hortas fiscalizadas.

Foi verificado que houve fraca correlação en tre a quantid ade de oocis tos de Crypto sporidium spp. e Esch erichia coli nas amostras de alface adquiridas em supermercado e feira livre. Foram utilizados os valores para o Cryptosporidium spp. obtidos com a utilização do método de coloração de Koster modificado, pois este apresentou melhor eficácia na detecção deste pro tozoário.

Mar zochi (1977) relata que a contaminação dos produtos vegetais por enteroparasitas depen de da con cen tração de matéria orgânica de ori gem fecal nas águas de irrigação proven ientes da dren a gem de esgotos domésticos. Mon ge et al. (1996), analisando hortaliças consumidas cruas em Costa Rica, verificaram correlação 
positiva entre a ocorrência de parasitas intestinais ecoli formes fecais.

$\mathrm{Na}$ avaliação da qualidade microbiológica de água e alimen tos incluindo as hortaliças, as bactérias do grupo coliformes são utilizadas como micror ganismos indicadores, portanto a pre sença de coli formes fecais su gere a presença de patógenos intestinais, porém Harich et al. (1999) citam que os coliformes não são bons indicadores para protozoários patogênicos, s endo de fundamental importância sua pesquisa em alimentos.

Considerando-se os re sult ados obti dos neste estudo, bem como o risco à saúde que as hortaliças podem apresentar quando contaminadas por parasitas intestinais, ressalta-se a necessidade da adoção de medidas que propiciem uma melhoria do quad ro apresentado, através de ações educativas destinadas aos produtores e do monitoramento laboratorial das águas destinadas à irrigação das hortas.

A desinfecção das hortaliças, previamente ao consumo, pode apres entar relevância con siderável no sentido de minimizar os riscos de transmissão de en teropara si toses através desses alimentos, uma vez que a lava gem simples não reduz a contaminação por cistos. Um método simples e eficaz consiste na imersão das folhas em água aquecida a $60^{\circ} \mathrm{C}$, por dez minutos (Oliveira \& Germano, 1992b). Este procedimento, pela maior facilidade de execução no nível doméstico, deve ser especialmen te considerado na formulação de programas educativos direcionados à população consumidora desses alimen to $\mathrm{s}$.

\section{Con clusões}

A ocorrência de oocistos de Cryptosporidium $s p p$. nas amostras de alface lisa vem alertar sobre o risco de infecção pela população humana, podendo provocar graves danos à saúde. Os demais enteroparasitas, coliformes totais e $E$. col $i$ foram en con trados em todas as variedades de hortaliças estudadas, comprovan do assim a necessidade de ações voltadas para a melhoria da qualidade na produção das hortaliças como da água utilizada na irrigação.

\section{Colaboradores}

Tese de mestrado do Programa de Pós-Graduação em Nutrição, área Ciência dos Alimentos, desenvolvida por CGMSi lva, sen do a estatística do trabalho re alizada pela professora SAC Andrade, sob a orientação da professora TLM Stamford.

\section{Agradecimentos}

Ao Laboratório de Experimentação e Análise de Alimentos (LEAAL), do Departam en to de Nutrição/UFPE, pelo consentimento da utilização dos equipamentos, e à Coordenação de Aperfeiçoamento de Pessoal de Nível Superior (Capes) pela bolsa de mestrado con cedida à primeira autora. 
Referências bibliográficas

And rews WH 1993. Food microbiology-nondairy. Journal of Association Official Analytical Chemists In ternational 76(1):154-159.

Bennet JW, Gauci MR, Moënic SL, Scraefer FW \& Lind quist HAD 1999. A comparis on of enu meration techniques for Cryptosporidium parvum oocysts. Journal of Parasitology 85(6):1165-1168.

Cimerman S, Ci m eman B \& Lewi DS 1999. Avaliação da relação en tre para si tosesinte s tinais e fatores de ris co p a ra o HIV em paci ien tes com Aids. Revista da Sociedade Brasilei rade Med icina Tropical 32(2):181-185.

Coelho LMPS, Oliveira SM, Milman MHSA, Karasawa KA \& San tos RP 2001. Detecção de formas transmissíveis de enteroparasitas na água e nas hortaliças con sumidas em comunidades esco $l$ a res de So rocaba, São Paulo, Brasil. Revista da Sociedade Brasileira de Medicina Tropical 34(5):479-482.

Freitas MAR, Vianna EM, Martins AS, Silva EF, Pesqu ero JL \& Gomes MA 2004. A Single Step Du pl exPCR to distinguish Entamoeba histolytica from Entamoeba díspar. Parasitology 128:625-628.

Gelli DS, Tachibana T, Ol ivei ra IR, Zamboni CQ, Pacheco JÁ \& Spiteri N 1979. Condições higiênico-sanitárias de hortaliças comercializadas na cidade de São Paulo, SP, Brasil. Revista do In s ti tuto Adolfo Lutz 39:37-43.

Gomes MA, Pesqu ero JB, Fu rst C, Valle PR, Pesqu ero JL \& Si lva EF 1999. An improved method to distinguish Entamoeba histolytica and Entamoeba díspar. Parasitology 119:359-362.

Guilherme ALF, Araújo SM, Falavigna DLM, Pupulim ART, Dias MLGG, Olivei ra HS et al. 1999. Prevalência de enteropa rasitas em horticultores e hortaliças da Feira do Produtor de Maringá, Paraná. Revista da Soci edade Brasilei ra de Medicina Tropical 32(4):405411.

Hari ch EM, G a lvani AT, Padula JA, Santos AIP, Men egon N \& Sa to MIZ 1999. Detecção de oocis tos de Giardia e oocistos de Cryptosporidium em águas su perficiais captadas para con sumo humano, pp. 27. XX Congres so Brasilei ro de Mi crobiologia, Bahia.

Kageruka P, Brand J, Taelman H \& Jonas C 1984. Mod ified Koster staining method for the diagnosis of cryptosporodiosis. Am erican Society Belge of Medicine Tropical 64:171-175.

Kori chDG, Me ad JR, Madore MS, Sin clair NA \& Sterling CR 1990. Effect of ozone, chorine dioxide, chlorine and monochloroamine on Cryptosporidium oocysts viability. Appliedand Envirnmental Mi crobiology 56: 1423-1428.

Lima EC 2001. Avaliação de métodos de deteç̧ão e enumeração de oocistos de Cryptosporidium spp. em amostras de água e leite. Dissertação de mestrado. Programa de Pós-Graduação em Nutrição, Un ivers i$\mathrm{d}$ ade Federal de Pernambuco, Recife.

Lima EC \& Stamford TLM 2003. Cryptosporidium spp. no ambiente aquático: aspectos relevan tes da disseminação e diagnósti co. Ciência e Saúde Coletiva 8(3): 791-800.
Luna LG 1968. Manual of histologic staining methods of the Armed Forces Institute of Pathology. 3a ed. McGraw Hill Book Com p a ny.

Marzochi MCA 1977. Estudo dos fatores envolvidos na disseminação dos enteroparasitas. II - Estu do da contaminação de verdu ras e solo de hortas na cidade de Ri beirão Preto, São Paulo, Brasil. Revista do In stituto de Med i cina Tropical de São Paulo 19:148-1556.

Mesquita VCL, Serra CMB, Bastos OMP \& Uchôa CMA 1999. Contaminação por enteroparasitas em hortaliças comercializadas nas cidades de Niterói e Rio de Ja n ei ro, Brasil. Revista da SociedadeBra sileira de Medicina Tropical 32(4):363-366.

Monge R \& Arias ML 1996. Presencia de microorganismos patógenos em hortalizas de con sumo crudo en Costa Rica. Archivos Latinoamericanos de Nutricion 46(4):292-294.

Mon ge R, Chinchilla M, Reyes L 1996. Estacionalidadde parásitos y bacterias inte stinales en hortalizas que se con su men crudas en Costa Rica. Revista de Biología Tropical 44(2):369-375.

Oliveira CAF \& Germano PML 1992a. Estudo da ocorrência de enteroparasitas em hortaliças com erciali$\mathrm{z}$ adas na região metropolitana de São Paulo, SP, Brasil. I - Pesquisa de helmintos. Revista de Saúde Pública 26(4):283-289.

Oliveira CAF \& Germano PML 1992b. Estudo da ocorrência de enteroparasitas em hortaliças com erciali$\mathrm{z}$ adas na região metropolitana de São Paulo, SP, Brasil. II - Pe s quisa de Pro tozoários intestinais. Revista de Saúde Pública 26(5):332-335.

Ongerth JE \& Stibbs HH 1987. Identification of Cryptosporidium ooc ysts in river water. Applied and Environmental Mi crobiology 53(4):672-676.

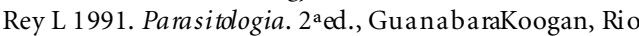
de Ja nei ro.

Silva CGM, Ol iveira AM \& Stamford TLM 2002. Enteroparasitas em vegetais: Uma revisão. Revista Higiene Alimentar 17(109):13-18.

Si lva JP, Ma r zochi MCA, Camilo-Coura L, Messias AA \& Ma rques S 1995. Estu do da contaminação por enteropa rasitas em hortaliças com ercializadas nos superm erc ados da cidade do Rio de Ja n ei ro. Revista da Sociedade Bra silei rade Med i cina Tropical 28:237-241.

Slifko TR, Smith HV, Rose JB 2000. Emerging parasite zoonoses associated with water and food. International Journal for Parasitology 30:1389-1393.

Tak ayanagui OM, Febrônio LHP, Ber gamini AM, Okino MHT, Silva AAMC CE, Santiago R, et al. 2000. Fiscalização de hortas produtoras de verduras do município de Ribeirão Preto, SP. Revista da Sociedade Brasilei ra de Med i cina Tropical 33(2):169-174.

Takayanagui OM, Oliveira CD, Bergamini AMM, Capuano DM, Okino MHT, Febrônio LHP et al. 2001. Fiscalização de verduras do município de Ribeirão Preto, SP. Revista da Soci edade Bra silei radeMedicina Tropical 34(1):37-41.

Artigo apresentado em 19/08/2004

Aprovado em 24/05/2005

Versão final apresentada em 22/06/2005 